\title{
Enhanced Water Dispersibility of Discrete Chalcogenide Nanoclusters with Sodalite-Net Loose-Packing Pattern in Crystal Lattice
}

Chaozhuang Xue,,$^{\dagger}$ Li Zhang, ${ }^{\dagger}$ Xiang Wang, ${ }^{\dagger}$ Dandan Hu, ${ }^{\dagger}$ Xiao-Li Wang, ${ }^{\dagger}$ Jiaxu Zhang, ${ }^{\dagger}$ Rui Zhou, ${ }^{\dagger}$ Dong-Sheng Li, ${ }^{\ddagger}$ Haifeng Su, ${ }^{\S}$ Tao $\mathrm{Wu}^{*}, \dagger$

†College of Chemistry, Chemical Engineering and Materials Science, Soochow University, Suzhou, Jiangsu 215123, China.

¥College of Materials and Chemical Engineering, Hubei Provincial Collaborative Innovation Center for New Energy Microgrid, Key Laboratory of Inorganic Nonmetallic Crystalline and Energy Conversion Materials, China Three Gorges University, Yichang, Hubei 443002, China

\$State Key Laboratory for Physical Chemistry of Solid Surfaces, Collaborative Innovation Center of Chemistry for Energy Materials, National \& Local Joint Engineering Research Center of Preparation Technology of Nanomaterials, College of Chemistry and Chemical Engineering, Xiamen University, Xiamen, Fujian 361005, China

*E-mail:1wutao@suda.edu.cn 


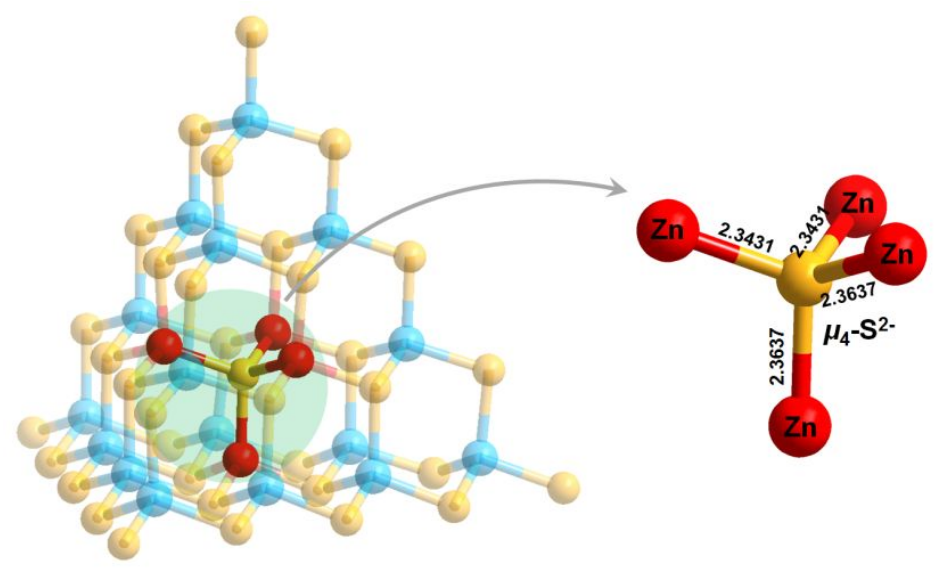

Figure S1. $\mu_{4}-\mathrm{S}^{2-}$ site at the core of supertetrahedral T4-ZnInS cluster.
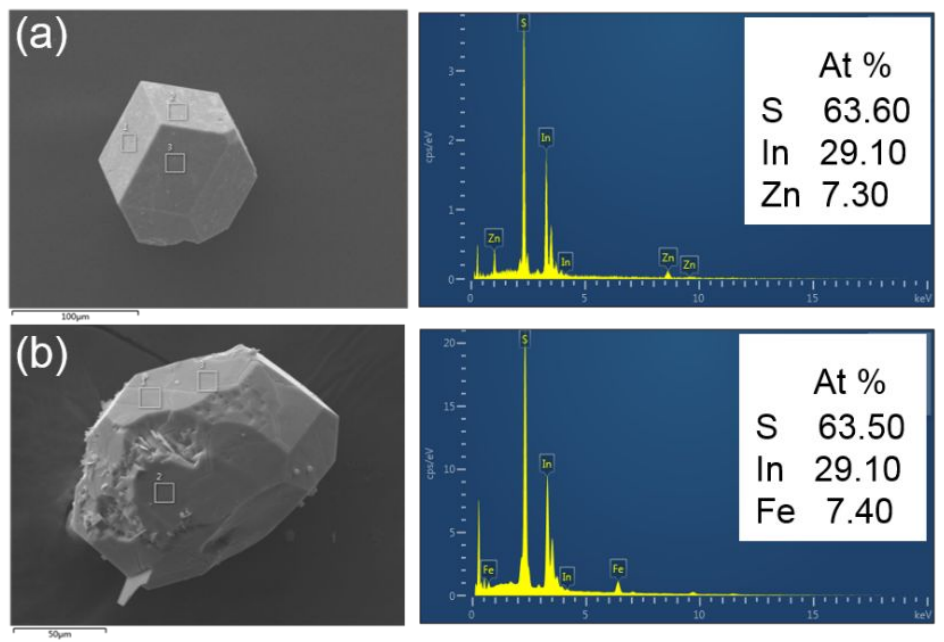

Figure S2. Energy dispersive spectra (EDS) of (a) ISC-16-ZnInS and (b) ISC-16-FeInS. 


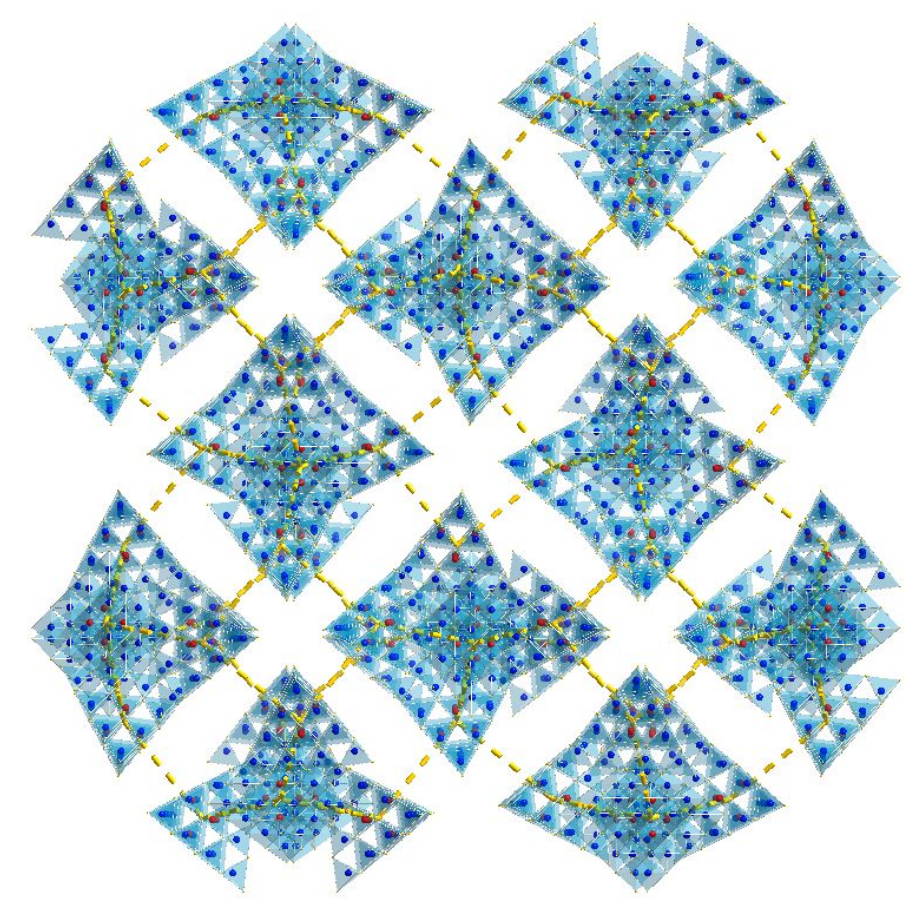

Figure S3. The sodalite-type framework of ICS-16 through the packing of isolated T4 subunits viewed from $b$-axis.
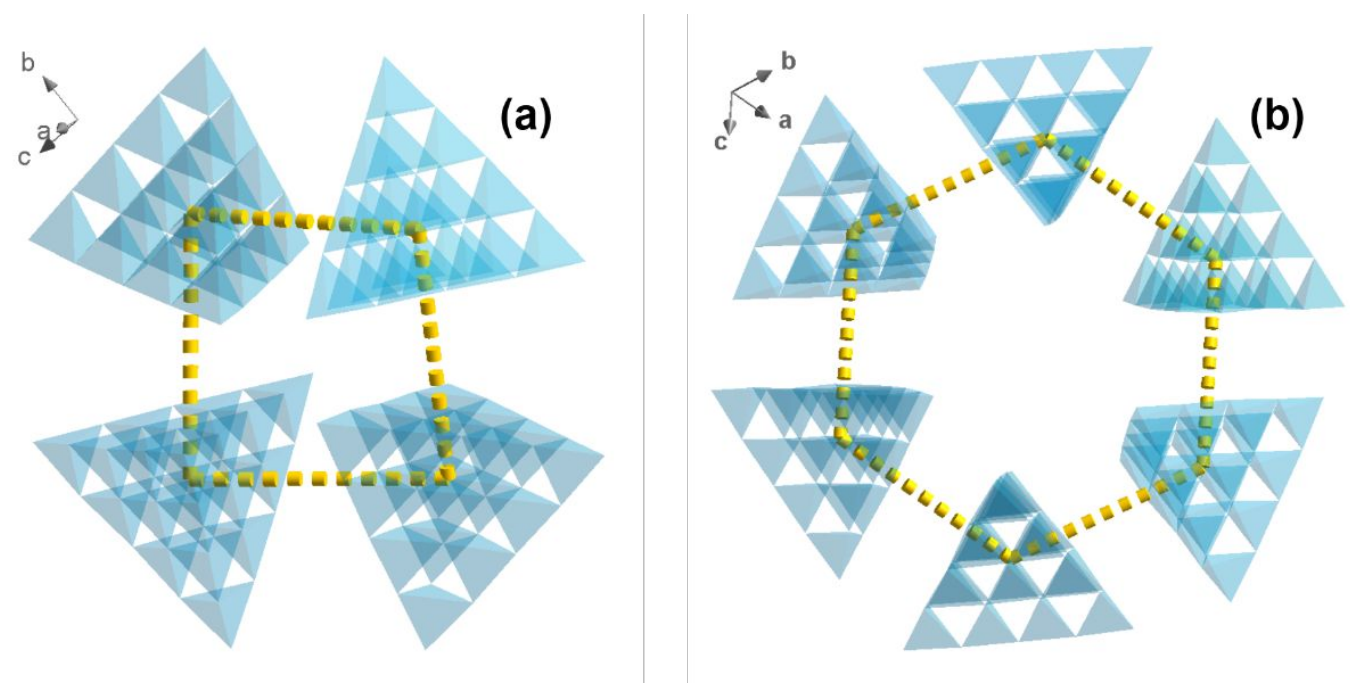

Figure S4. Two kinds of windows in SOD cage of ISC-16 : (a) four-membered rings of window A and (b) six-membered rings of window B. 


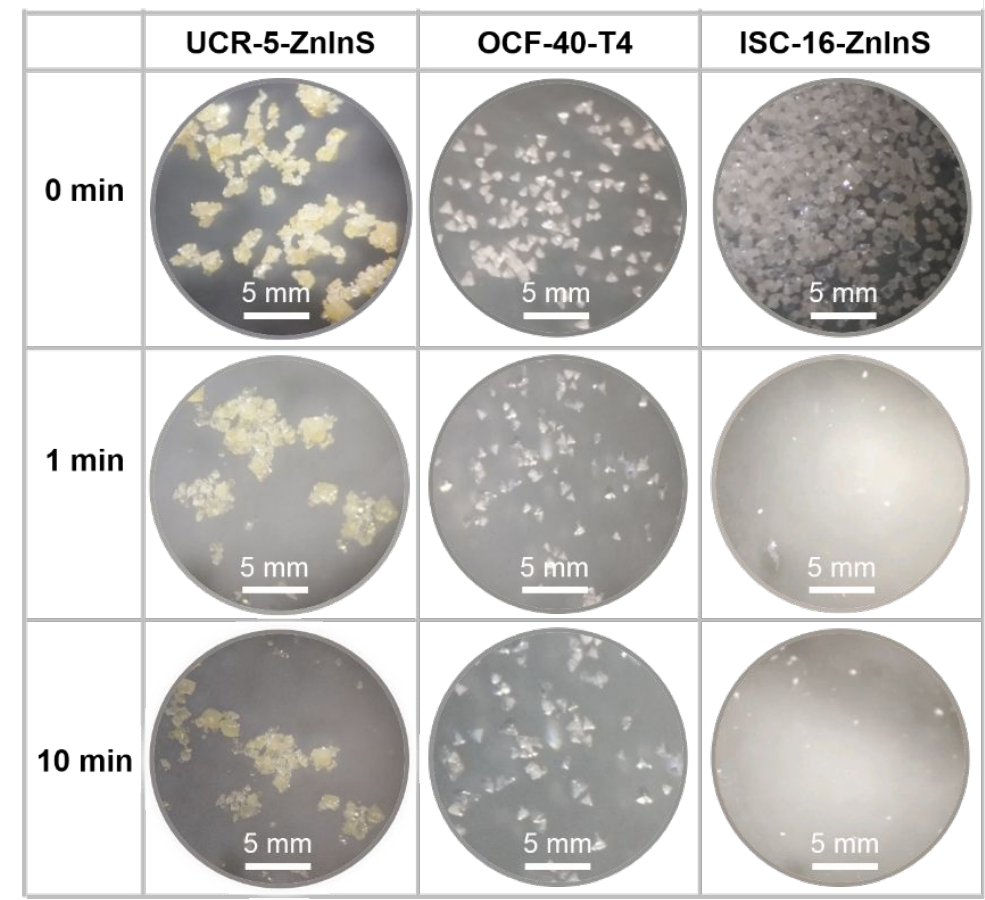

Figure S5. Dispersibility measurements on three T4 cluster-based chalcogenide materials.
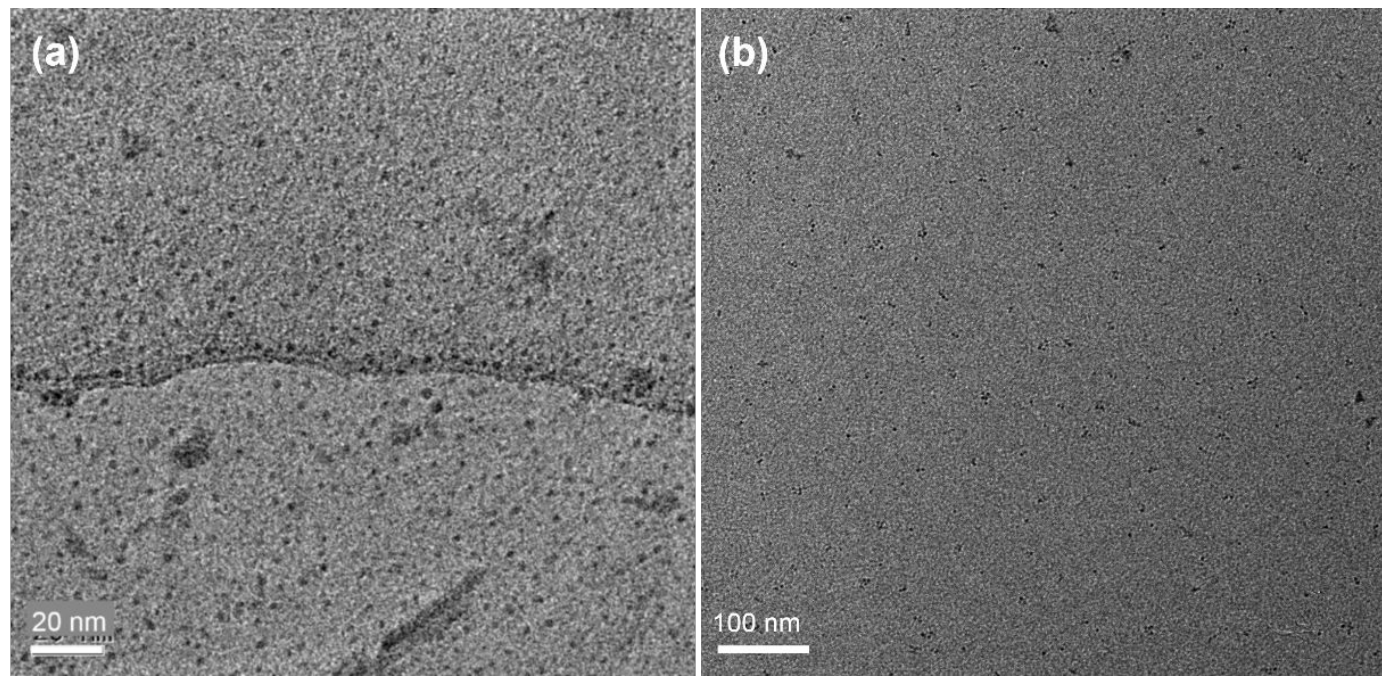

Figure S6. TEM image of the cluster-aggregated nanoparticles dispersed in piperidine solvent: (a) dispersed ISC-16-ZnInS particles; (b) dispersed ISC-16-FeInS particles. 

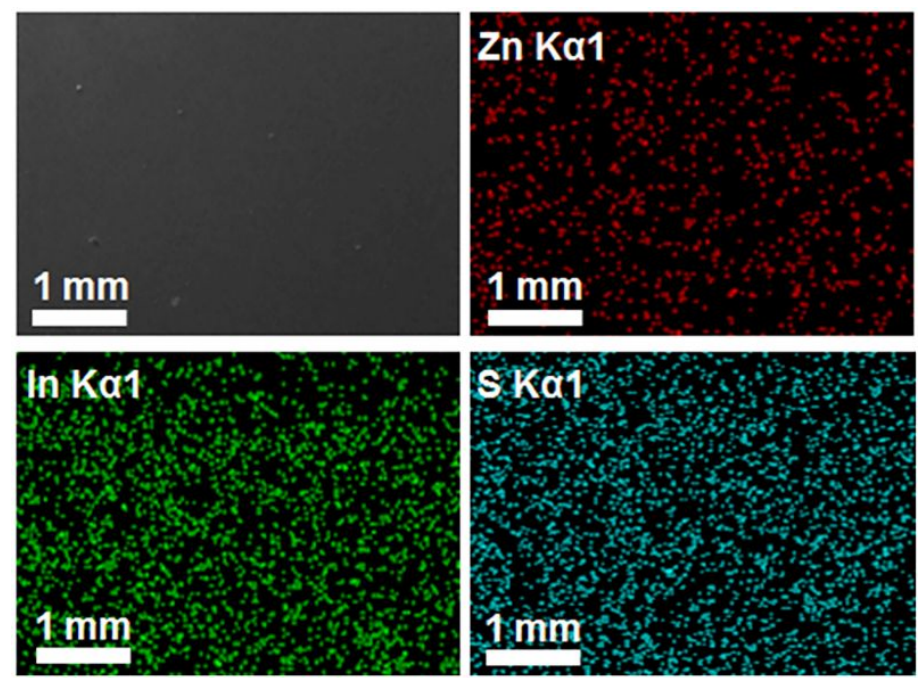

Figure S7. Elemental mapping for the dispersed T4-ZnInS cluster of ICS-16.

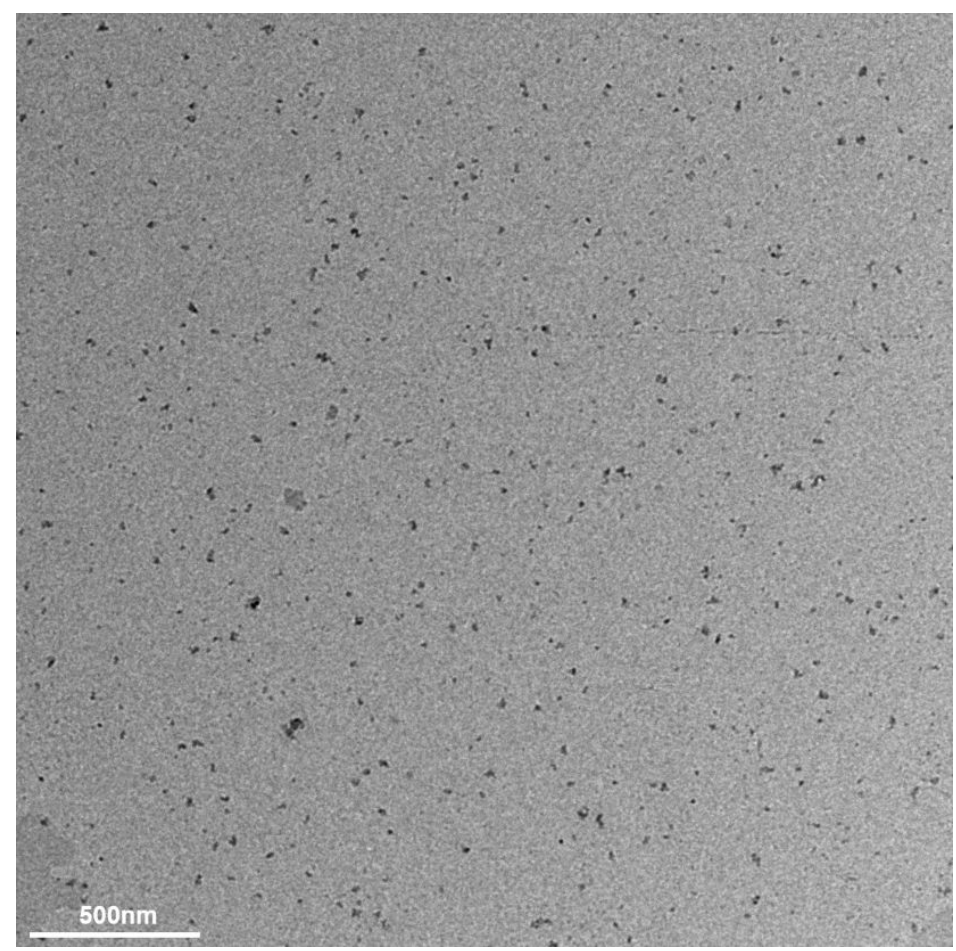

Figure S8. TEM image of the cluster-aggregated nanoparticles (ICS-16-ZnInS) dispersed in water. 


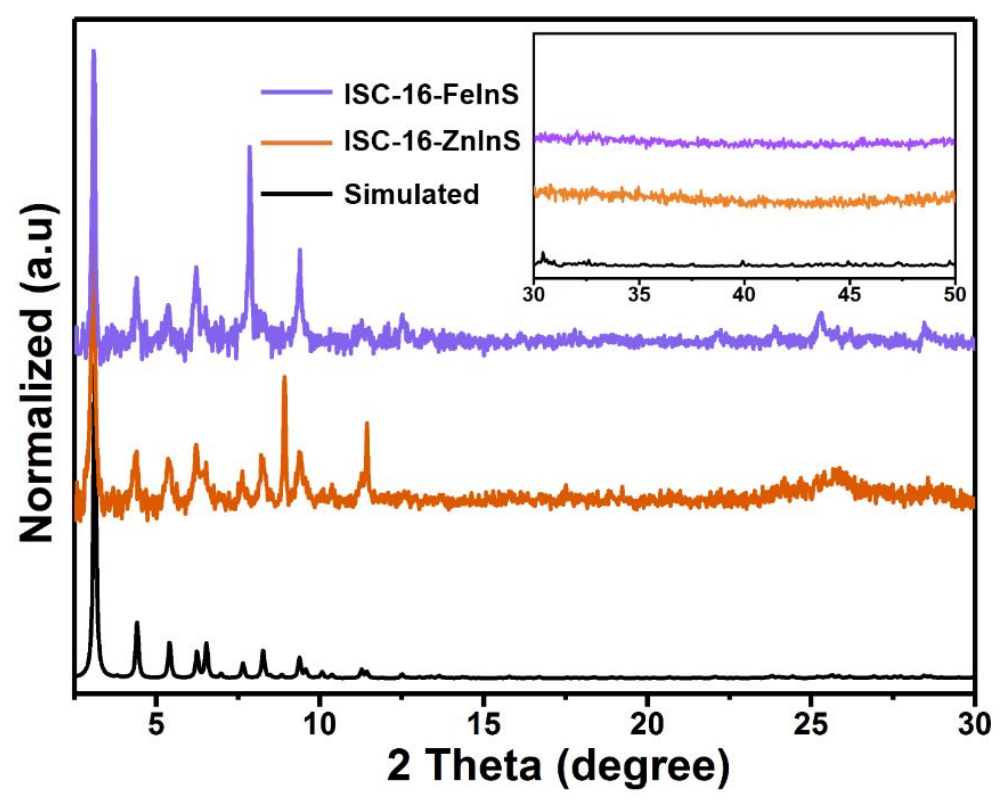

Figure S9. PXRD patterns of as-synthesized ISC-16-MInS. No obvious diffraction peaks of ISC-16-MInS can be observed at high degree (inset picture).
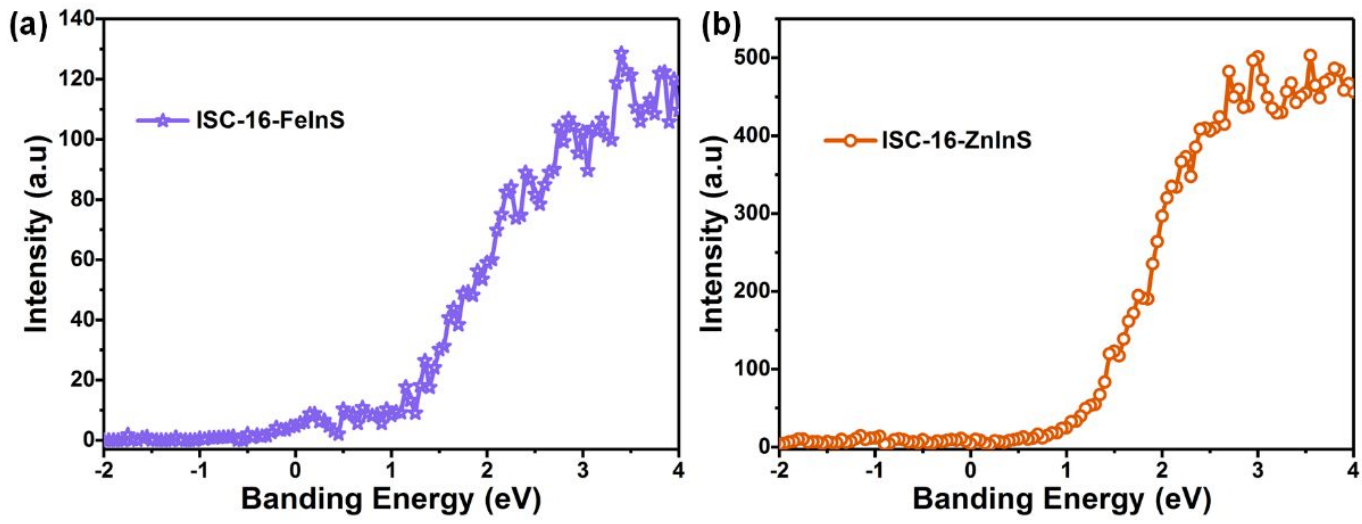

Figure S10. Valence band spectra of (a) ISC-16-FeInS and (b) ISC-16-ZnInS. 
(a)

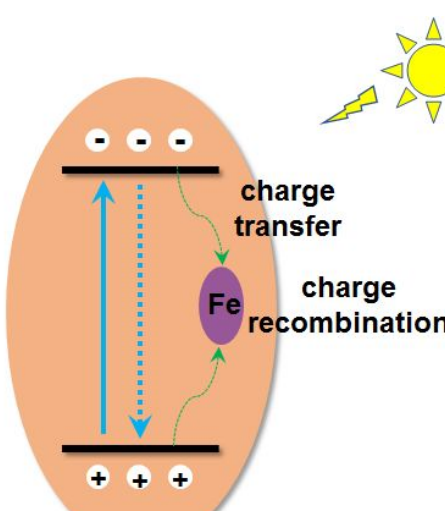

(b)

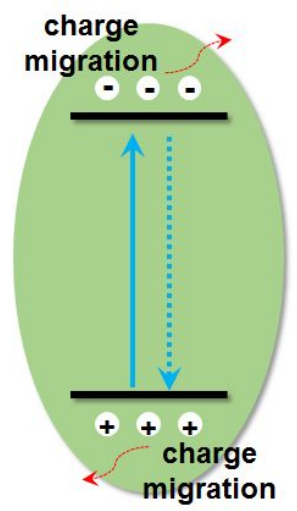

Figure S11. Scheme for the photogenerated charge transfer and migration in (a) ISC-16-FeInS and (b) ISC-16-ZnInS. 
Table S1. Crystal data and structure refinement parameters for ISC-16-ZnInS (The crystal data for ISC-16-FeInS is not available due to its poor diffraction data).

\begin{tabular}{|c|c|}
\hline & ISC-16-ZnInS \\
\hline Framework Formula & {$\left[\mathrm{Zn}_{4} \operatorname{In}_{16} \mathrm{~S}_{35}\right]$} \\
\hline Formula weight & 3220.70 \\
\hline Crystal morphology & dodecahedron \\
\hline Crystal system & Cubic \\
\hline$Z$ & 96 \\
\hline Space group & $F d-3 c$ \\
\hline$T / \mathrm{K}$ & $296(2)$ \\
\hline$\lambda / \AA$ & 0.71073 \\
\hline$a / \AA$ & $79.315(2)$ \\
\hline$b / \AA$ & $79.315(2)$ \\
\hline$c / \AA$ & $79.315(2)$ \\
\hline$a /{ }^{\circ}$ & 90 \\
\hline$\beta /{ }^{\circ}$ & 90 \\
\hline$\gamma / \mathrm{o}$ & 90 \\
\hline$V\left(\AA^{-3}\right)$ & $498960(38)$ \\
\hline$F(000)$ & 140544 \\
\hline$D\left(\mathrm{~g} \mathrm{~cm}^{-3}\right)$ & 1.029 \\
\hline$\mu\left(\mathrm{mm}^{-1}\right)$ & 2.542 \\
\hline Collected reflections & 3955 \\
\hline Independent reflections & $2513\left(R_{\mathrm{int}}=0.1644\right)$ \\
\hline GOF on $F^{2}$ & 1.058 \\
\hline$R_{1}, w R_{2}(I>2 \sigma(I))$ & $0.0700,0.1697$ \\
\hline$R_{1}, w R_{2}$ (all data) & $0.1036,0.2136$ \\
\hline
\end{tabular}

Alert level A: The value of sine(theta max)/wavelength is less than 0.550 Calculated $\sin ($ theta_max $) /$ wavelength $=0.3568$

Response: The diffraction capability of cluster-based chalcogenide is usually weak, especially for the structures that are constructed by isolated clusters, and it's hard to harvest high-angle diffraction points. We cut off bad diffraction points at high resolution to improve the data quality. 